\title{
ADVANCED KEYLOGGER FOR ETHICAL HACKING
}

\author{
Sarita Yadav, Anuj Mahajan, Monika Prasad, Avinash Kumar \\ Department of IT \\ BVCOE, New Delhi, Delhi, India
}

\begin{abstract}
Data Security professionals work each day taking care of security issues and taking care of dangers. These specialists attempt to keep away from new security dangers, however, the attackers are attempting to discover new infiltration strategies and refined assaulting techniques to discredit PCs. The quantity of these attackers is expanding in the PC world today. The utilization of keylogger is being utilized for distinguishing and logging what intruders are doing when performing advances. The keylogger can log the entered keystrokes on hosts, for example, remote frameworks and in honeypots. Accumulating keystrokes is a significant advance towards getting programmers and obtain information about the advances. The keylogger can record all exercises identified with the accentuation on the console keys or action on the working framework. String coordinating is a strategy for coordinating characters from the info procedure like a console, and this coordinating procedure is finished by confirming each approaching character. The utilization of string-coordinating calculations on keylogger applications used to screen client action makes it increasingly agreeable and quicker.
\end{abstract}

\section{INTRODUCTION}

Malware is named by various names, Such as pernicious code, malignant programming. Numerous characterize malignant code as "any code included, adjusted, or erased from a product framework to deliberately cause hurt or destabilize the planned capacity of the framework". Key loggers [21] are getting progressively varied, repulsive, current, and progressively hard to identify by anti-virus and against keyloggers dependent on signature examination.

Keylogger is a malware [12] that tracks the client's composed keystroke on the console. The goal of the keylogger is to covertly record classified data of the client's contribution through keystroke observing [8] and afterward passing this significant data to other people. The console is the central technique for contributing printed and numerical data on the PC through creating. Ordinarily, there is no knowledge achieved in keylogger, however, logs offer data about every console occasion and applications that clients clicked or composed. Regardless of the absence of data on what application is utilized, $\operatorname{logs}$ give enough data that permits one to comprehend what clients are doing. Information caught incorporate passwords, client IDs, archive substance, and other basic data [2]. Keylogging is imperceptible as it runs in secrecy mode. These keyloggers can't be distinguished by numerous Anti-Malware programs running on the device. The client has no real way to recognize the nearness of keyloggers on his device.

\section{Motivation}

This area is about the inspiration for this project, keylogger, and significance around that subject. All the accompanying articles contain distinctive virtual advancements [11][18][19], assaults that are referenced beneath give enthusiasm for making a proposition of issue proclamations to comprehend. There are two fascinating cases around the subject keylogger, found in papers on the Internet. One study from the year 2005 and the other from 2014, that bestows that keyloggers are utilized for quite a while now.

In February 2005, Joe Lopez, an agent from Florida, documented a suit against Bank of America later unknown programmers took 90,000 Dollars from his Bank of America account.

An examination indicated that Mr. Lopez's PC was tainted with a wicked program. Coreflood, which records each key stroke and transmits this data to harmful clients utilizing the Internet. This is the way the programmers got hold of Joe Lopez's client name and secret word.

During February 2014 there was an article in www.nrk.no which read that the Norwegian Police Security Service (PST) approach lawmakers for consent to introduce approaches to screen information consoles of individuals they have at the center of attention. This could be accomplished by introducing a legitimate keylogger covertly on the distant computer to log keystrokes.

Keylogging has grown a built-up apparatus utilized by aggressors for obtaining passwords and other classified information. For programmers, yet besides for others, for example, departmental heads for frameworks, recognizing dubious movement [4][6]. In investigating, for various territories, for example, for a look into by guardians for checking their youngsters and recognizing uncommon practices and to distinguish lawbreakers. Keyloggers can likewise be a helpful apparatus to distinguish assaults and their instruments. 


\section{International Journal of Engineering Applied Sciences and Technology, 2020 Vol. 5, Issue 1, ISSN No. 2455-2143, Pages 634-638 \\ Published Online May 2020 in IJEAST (http://www.ijeast.com)}

The inspiration for this venture is to discover whereby keylogging functions under various advances and set up a honeypot to log the keystrokes, entered as orders by the clients. With the reason to survey precisely what the clients are doing. This instrument will screen which strategy will be utilized. This may likewise prompt fruitful cooperation with the programmer, to identify keystrokes that may get ready against such assaults later on.

\section{SOLUTION APPROACH}

In all exchange businesses nowadays, work areas and IT divisions are a monster and reveling framework. Representatives in all divisions from HR to program progress anticipate a figuring gadget as well as system connector to carry out their responsibilities easily. Indeed, even the individuals in the field are expected to hold a work area or a few types of handheld gadgets to transmit data. This way to deal with the field has presented an amount of ongoing assurance related subject to the work drive. One of the significant issues incorporates the producer's business to keep authority over InfoTech and instructive organizations assets which give staff a chance to quietly execute their private occasions. There are over a hundred altogether unforeseen measurements these days that may let associations notice what their representatives demonstrate at the particular employment on their work areas, of their email and on the web [20]. In any case, what do such insights decently speak to? What do associations watch out for client/worker electronic message, web, and PC usage genuinely resemble? You have to screen your representatives, the idea of keyloggers is fundamentally significant. This keylogging venture catches and records all keys where the keystrokes had been squeezed. Utilizing this, we hold onto all information in literary substance.

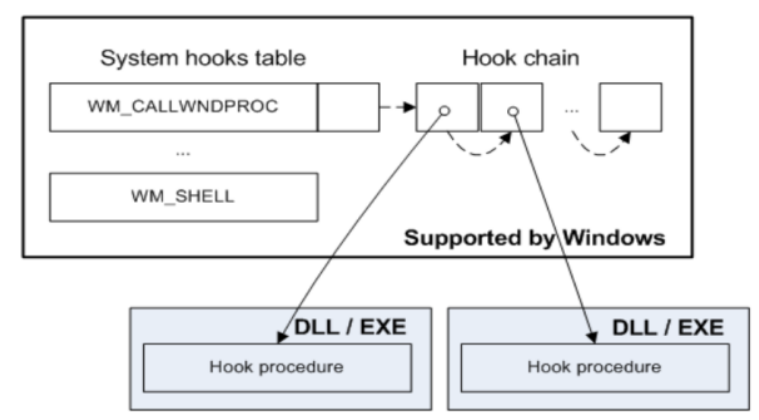

Figure 1: Client-Server Method

A console comprises a network of paths overlapped including keys. This grid of circuits, identified as a key matrix, can contrast between console makers. Nonetheless, the important codes that are transmitted by the console interface to a particular working framework are consistently the equivalent.

At the point when the client presses a key, a circuit shuts in the Fundamental Matrix [10]. The keyboard distinguishes this occasion and catches the circuit area. Utilizing a record put away in console ROM, the processor interprets the circuit area to a character or control code. Key codes are regularly CTRLor ALT-mixes.

The console's memory support incidentally stores the deciphered character or control code and afterward sends it to the PC's console interface. The console controller gets the approaching console information and advances it to the working framework. A console driver is regularly practiced to deal with this piece of the procedure. The working framework forms the console information dependent on the present condition of the Operating System and programs.

Some principal techniques to create keylogger frameworks: The "Windows Keyboard Hook" strategy, the "Keyboard State Table" strategy, and the "Kernel-Based Keyboard" [9]. Initially, the Windows Keyboard Hook strategy dependent on the OS that gives a few capacities to Hook-based keyloggers for checking the console. At the point when a key is squeezed the Operating System records the activity and registers the application itself. Following all information moving in this instrument is affirmed by the application before heading off to the first objective that gets the message. Our keylogger uses these same methods to catch key strokes.

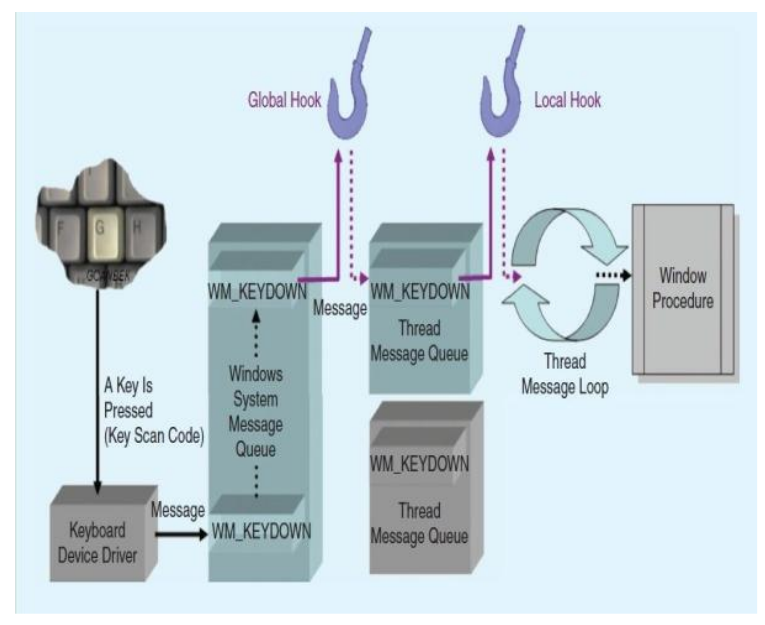

Figure 2: Global Hook vs Local Hook

Some particular kinds of hooks identified with windows communication: Global hook controls framework broad information and local hook screen application-explicit message. Console hooks are:

1) Competent in perusing all console messages and move them into the following hook strategy in a series.

2) Ready to change the initial information and relinquish it to the following hook method.

3) Capable to intrude on the progression of the information by not transferring it to the following hook strategy.

An equipment keylogger can be spotted easily but difficult to spot if a client reviews everything that is associated with a console to the equipment on a PC, however, programming keyloggers are progressively hard to recognize, in light of the 


\section{International Journal of Engineering Applied Sciences and Technology, 2020 \\ Vol. 5, Issue 1, ISSN No. 2455-2143, Pages 634-638 \\ Published Online May 2020 in IJEAST (http://www.ijeast.com)}

fact that they are programs inside a PC. A decent component to this and any keylogger implies it remains imperceptible and difficult to recognize in the present framework. Particularly when this design is to stow away the keylogger for the clients.

\section{CONCLUSION}

We read about several techniques of how the data is transferred from any client in encrypted form and implemented the Base 64 encryption technique [1][3]. The applications of keyloggers for various purposes and in different sectors of society is understood and taken into account in making of this project.

To start the keylogger just start the executable file this is the only file that is required for this keylogger to work.

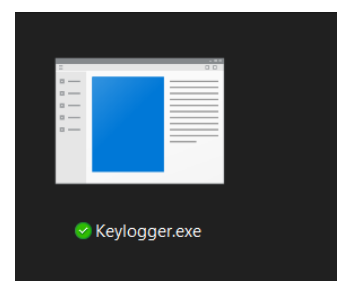

Figure 3: Keylogger Executable

Existing techniques can fail against advanced keyloggers and how any regular keylogger is detected by any Anti-Virus or Anti-Malware applications. Therefore, an additional effort has been made to make the keylogger stealth and hidden from the user and all types of Anti-virus.

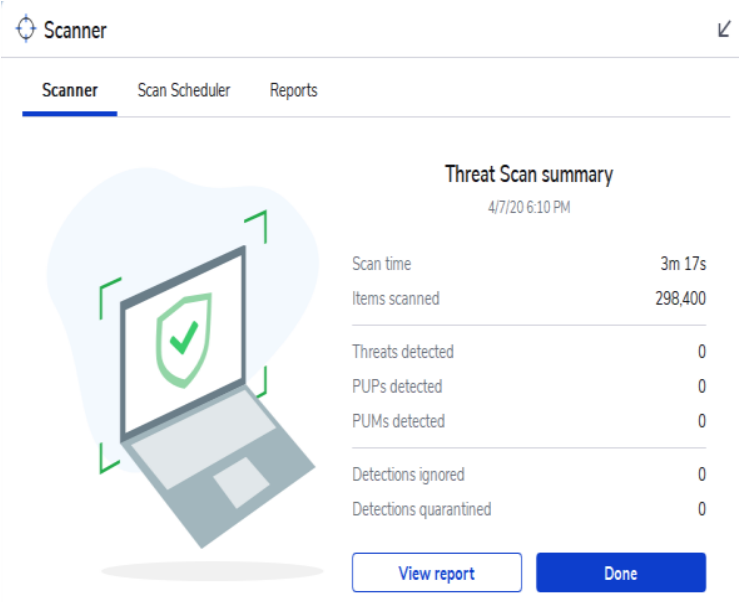

Figure 4: Anti Virus Scan

Even by manually checking the Task manger this program shows up like a default windows application.

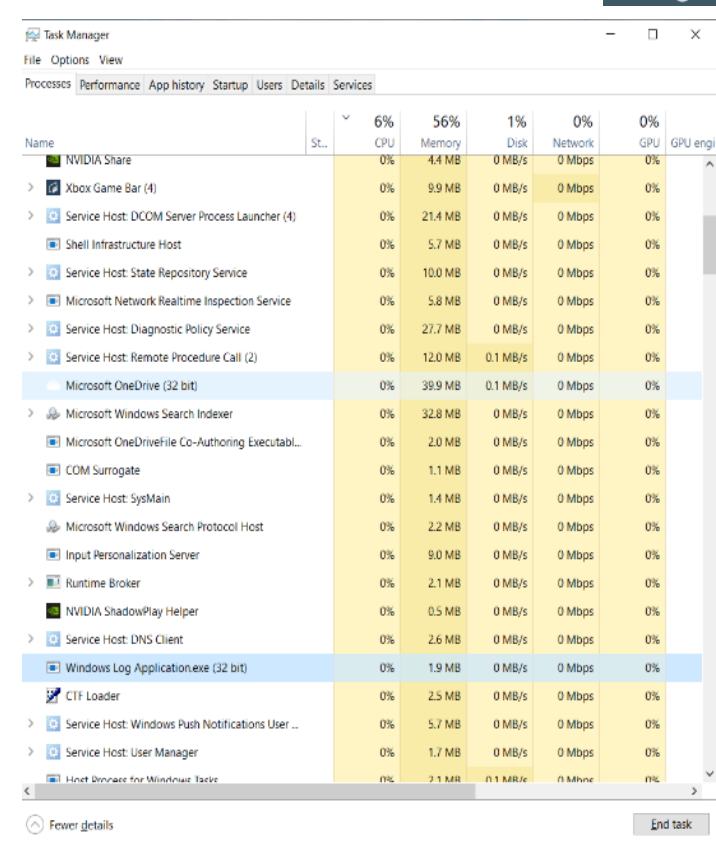

Figure 5: Keylogger in Task Manager

This Keylogger records the keystrokes pressed on a Windows platform. It stores them locally on the host system. Even if this file is detected by user it is stored in Encrypted format [14] as seen below using Base 64.

\author{
07.04.2020 18_15_46.log - Notepad \\ File Edit Format View Help \\ XLzovXzMc3bGluzT13b3dDKytMTTo6VEI60kJC
}

Figure 6: Logs on Host Machine

The $\operatorname{logs}$ are mailed in this same format so they are undetectable [7] by any user unless decrypted. For testing and debugging purposes the executable creates a file named AppLog.txt stored locally that help us to keep record if the process was successful or not, if any error occurs, we can detect it and solve using this log file. The content of log file are as follows: 
International Journal of Engineering Applied Sciences and Technology, 2020

Vol. 5, Issue 1, ISSN No. 2455-2143, Pages 634-638

Published Online May 2020 in IJEAST (http://www.ijeast.com)

Applog.txt - Notepad

File Edit Format View Help

|[07.04.2020 18:14::2]

Hook started... Timer started

[07.04.2020 18:14:39]

Mail was not sent! Error code: 259

[07.04.2020 18:14:39]

〈From SendMail〉 Return Code: 0

[07.04.2020 18:15:16]

Mail was not sent! Error code: 259

[07.04.2020 18:15:53]

Mail was not sent! Error code: 259

Figure 7: Applog on Host machine

These error codes would help if the log was not sent due to any reason. The $\log$ files are encrypted and decrypted to provide and additional layer of security. In scenarios where user manages to get his hands on the logs, he won't be able to understand it as the files are encrypted and can only be decrypted by the system administrator. this:

The mail that is received on the admin account looks like

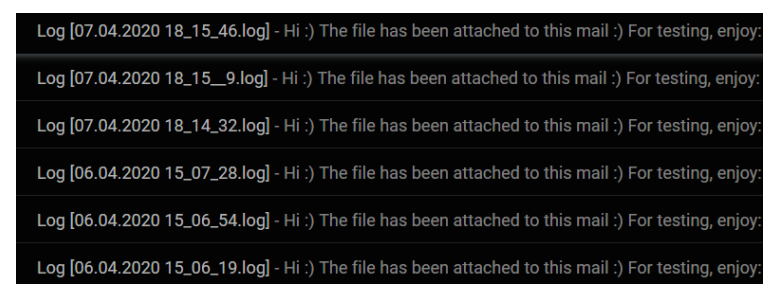

Figure 8: Mail as Received by admin

After decrypting the encrypted file received in the mail we can see the keystokes of the Host machine.
$H i$ )

\section{The file has been attached to this mail : \\ For testing, enjoy:

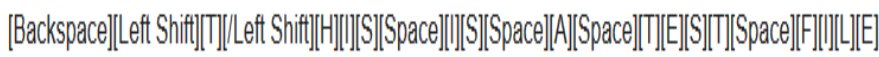

Figure 9: Decrypted logs

\section{REFERENCE}

[1] Rahim R. and Ikhwan A. (2016); Study of Three-Pass Protocol on Data Security International Journal of Science and Research (IJSR) 5 (pp. 102-104).

[2] Rahim R. (2017); 128 Bit Hash of Variable Length in Short Message Service Security International Journal of Security and Its Applications 11 (pp. 45-58).

[3] Siahaan A. P. U. and Rahim R. (2016); Dynamic Key Matrix of Hill Cipher Using Genetic Algorithm International Journal of Security and its Applications 10 (pp. 173-180).

[4] Venkatesh R. and Sekhar R. K. (2015); User Activity Monitoring Using Keylogger Asia Journal of Information Technology 15 (pp. 4758-4762).

[5] Soni K. K., Vyas R. and Sinhal A. (2014); Importance of String Matching in Real-World Problems International Journal of Engineering And Computer Science 3 (pp. 6371-6375).

[6] Adhikary N., Shrivastava R., Kumar A., Verma S. K., Bag M. and Singh V. (2012); Battering Keyloggers and Screen Recording Software by Fabricating Passwords I. J. Computer Network and Information Security 2012 (pp. 13-21)

[7] Dadkhah M., Jazi M. D., Ana-Maria C. and Barati E. (2014); An Introduction to Undetectable Keyloggers with Experimental Testing International Journal of Computer Communications and Networks 4 (pp. 1-5).

[8] Tuli P. and Sahu P. (2013); System Monitoring and Security Using Keylogger International Journal of Computer Science and Mobile Computing 2 (pp. 106-111).

[9] Tian, Donghai, et al. (2017); "An Online Approach for Kernel-level Keylogger Detection and Defense." J. Inf. Sci. Eng. 33.2: (pp. 445-461).

[10] Raiu, Costin, and Igor Soumenkov. (2015); "Comparing the Regin module 50251 and the" Qwerty" keylogger.": 2015.

[11] Santwana, C., K. Sai Aditya, and S. Magesh. (2015); "Hypervisor based Mitigation Technique for Keylogger Spyware Attacks." International Journal of Computer Science and Information Technologies.

[12] Hoglund, Greg, and James Butler. Rootkits (2006); subverting the Windows kernel. Addison-Wesley Professional, (2006).

[13] Acharya, Bibhudendra, et al. (2009); "Image encryption using advanced hill cipher algorithm." International Journal of Recent Trends in Engineering 1.1: (pp. 663-667). 
[14] Coppersmith, Don. (1994); "The Data Encryption Standard (DES) and its strength against attacks." IBM journal of research and development 38.3: (pp. 243-250).

[15] Teske, Edlyn. (1999); "The Pohlig-Hellman method generalized for group structure computation." Journal of Symbolic Computation 27.6: (pp. 521-534).

[16] Wang, Xiaoyun, et al. (2004); "Collisions for Hash Functions MD4, MD5, HAVAL-128 and RIPEMD." IACR Cryptology ePrint Archive 2004: 199.

[17] Raiu, Costin, and Igor Soumenkov. (2015); "Comparing the Regin module 50251 and the" Qwerty" keylogger.": 2015. [18] Bobbitt, Jared E., et al. (2006) "Virtual file system." U.S. Patent No. 7,024,427. 4 Apr.

[19] Uhlig, Rich, et al. (2005); "Intel virtualization technology." Computer 38.5: (pp. 48-56).

[20] Park, Dae-woo. (2016); "Analysis of Phising, Pharming and Smishing Spam Mail Trend and Techniques from Other Countries." International Information Institute (Tokyo). Information 19.3: 895

[21] Sagiroglu, Seref, and Gurol Canbek. (2009); "Keyloggers: Increasing threats to computer security and privacy." IEEE technology and society magazine 28.3: (pp. 10-17). 